\title{
CPU/GPU COMPUTING FOR AN IMPLICIT MULTI-BLOCK COMPRESSIBLE NAVIER-STOKES SOLVER ON HETEROGENEOUS PLATFORM
}

\author{
LIANG DENG \\ Computational Aerodynamics Institute, China Aerodynamics Research Development Center, Mianyang, \\ Sichuan, China \\ denglnudt@126.com \\ HANLI BAI \\ Computational Aerodynamics Institute, China Aerodynamics Research Development Center, Mianyang, \\ Sichuan, China \\ baihanli@163.com \\ FANG WANG \\ School of Computer, National University of Defense Technology, Changsha Hunan, China \\ wwangfang@sina.com \\ QINGXIN XU \\ Computational Aerodynamics Institute, China Aerodynamics Research Development Center, Mianyang, \\ Sichuan, China \\ xuqingxin@cardc.cn
}

Published 22 June 2016

\begin{abstract}
CPU/GPU computing allows scientists to tremendously accelerate their numerical codes. In this paper, we port and optimize a double precision alternating direction implicit (ADI) solver for three-dimensional compressible Navier-Stokes equations from our in-house Computational Fluid Dynamics (CFD) software on heterogeneous platform. First, we implement a full GPU version of the ADI solver to remove a lot of redundant data transfers between CPU and GPU, and then design two fine-grain schemes, namely "one-thread-one-point" and "one-thread-one-line", to maximize the performance. Second, we present a dual-level parallelization scheme using the CPU/GPU collaborative model to exploit the computational resources of both multi-core CPUs and many-core GPUs within the heterogeneous platform. Finally, considering the fact that memory on a single node becomes inadequate when the simulation size grows, we present a trilevel hybrid programming pattern MPI-OpenMP-CUDA that merges fine-grain parallelism using OpenMP and CUDA threads with coarse-grain parallelism using MPI for inter-node communication. We also propose a strategy to overlap the computation with communication using the advanced features of CUDA and MPI programming. We obtain speedups of 6.0 for the ADI solver on one Tesla M2050 GPU in contrast to two Xeon X5670 CPUs. Scalability tests show that our implementation can offer significant performance improvement on heterogeneous platform.
\end{abstract}

Keywords: Multi-block; structured grid; alternating direction implicit; CFD solver; MPI-OpenMPCUDA; CPU/GPU computing.

This is an Open Access article published by World Scientific Publishing Company. It is distributed under the terms of the Creative Commons Attribution 3.0 (CC-BY) License. Further distribution of this work is permitted, provided the original work is properly cited. 


\section{Introduction}

As CPU/GPU heterogeneous systems can achieve higher floating-point performance and lower power consumption than CPU-only systems, there are increasingly more GPUbased high-end supercomputers appearing in the TOP500 lists, such as $\operatorname{Titan}^{1}$ and Tianhe- $1 \mathrm{~A}^{2}$. Comparing with CPU-only systems, these heterogeneous processors with different processing capabilities, memory availability, and communication latencies ${ }^{3}$ lead to challenging problems in implementing scientific applications and obtaining the best performance. The performance of an application will depend on the application operation's data access and processing patterns. To design more efficient applications, researchers often need to understand the performance characteristics of classes of application operations and use various programming models for heterogeneous devices.

For CFD applications, CFD solver is normally categorized into explicit and implicit schemes. In general, an implicit scheme has the advantages of less computing cost and good calculation stability, is the major computing method of practical CFD applications. However, from the parallel computation point of view, an implicit scheme is difficult to implement CPU/GPU heterogeneous parallel because it has high data dependence.

In recent years, lots of CFD applications have achieved significant performance improvement on heterogeneous systems. From the many recent studies, researchers mainly focus on approaches suitable to data parallelism, such as explicit schemes and implicit Jacobi iteration. Corrigan et al. ${ }^{4}$ studied an explicit Runge-Kutta solver for threedimensional compressible Euler equations using a NVIDIA Tesla GPU and got roughly 95 performance over a quad-core CPU. Jespersen et al. ${ }^{5}$ accelerated Jacobi iteration section from the CFD code OVERFLOW using a GPU and shown a speedup by a factor between 2.5 and 3 compared to a single CPU. Griebel et al. ${ }^{6}$ implemented and optimized a two-phase solver for the Navier-Stokes equations using the Runge-Kutta time integration on a multi-GPU platform and achieved an impressive speedup of 69.6 on eight GPUs/CPUs. Jacobsen et al. ${ }^{7}$ utilized the MPI-CUDA programming pattern to implement a Jacobi iterative solver for the incompressible Navier-Stokes equations on the Lincoln GPU cluster with 128 GPUs and obtained a speedup of 130 over the CPU solution using Pthreads on two quad-core Intel Xeon processors. They also compare the scalability between the tri-level MPI-OpenMP-CUDA and dual-level MPI-CUDA implementations ${ }^{8}$, the weak scale efficiency of the implementations on 128 GPUs only achieved $17 \%$ and $19 \%$. However, they only used OpenMP to substitute intra-node MPI communication rather than collaborating CPU and GPU for computation. Xu et al. ${ }^{9}$ presented a new tri-level MPI-OpenMP-CUDA parallelization scheme to collaborate CPU and GPU for a complex, real-world CFD application using explicit Runge-Kutta solver on the Tianhe-1A supercomputer and achieved a speedup of about 1.3 when comparing one Tesla M2050 GPU with two Xeon X5670 CPUs and a parallel efficiency of above $60 \%$ on 1024 Tianhe-1A nodes.

In contrast, there have been few researches on the implementations and optimizations of ADI scheme on CPU/GPU heterogeneous platform for practical CFD applications. Sakharnykh et al. ${ }^{10,11}$ investigated the ADI scheme to simulate incompressible, viscid 
fluid flows on CUDA-enabled GPUs and obtained an about 9.5 times speedup compared to using a single CPU core. Zhang et al. ${ }^{12}$ presented a parallel ADI solver on GPUs, optimized inefficient data accesses by leveraging hardware texture memory and matrix transpose techniques and achieved overall more than 100 times speedup over a highly optimized CPU version. However, on one hand, the above studies only considered the ADI scheme section of the whole CFD solver, while the computation of inviscid flux terms and the computation of viscid flux terms were ignored. On the other hand, they simply implemented a naïve GPU-only approach and wasted the multi-core CPU capacity.

In this paper, we develop a tri-level MPI-OpenMP-CUDA parallelization scheme of a double precision ADI solver for three-dimensional compressible Navier-Stokes equations from our in-house CFD code, $\mathrm{TH}-\mathrm{CFD}^{13}$, on heterogeneous platform. This implementation can tap the full potential of heterogeneous platform including multi-core CPUs and many-core GPUs.

The paper is organized as follows. In the next section, we briefly describe the governing equation and the formulation of the ADI scheme. Section 3 details the CUDA implementation. Section 4 extends our solver to CPU/GPU heterogeneous platform with a mixed OpenMP-CUDA implementation. Section 5 presents the overall consideration for the tri-level MPI-OpenMP-CUDA parallelization scheme. Experimental results are presented and analyzed in Section 6. Finally, Section 7 concludes the paper.

\section{Governing Equations and Numerical Approach}

\subsection{Governing equations}

In curvilinear coordinate the three-dimensional Navier-Stokes equations for viscid, compressible flow in strong conservative form are

$$
\frac{\partial \hat{\boldsymbol{Q}}}{\partial t}+\left(\frac{\partial \hat{\boldsymbol{F}}_{c}}{\partial \xi}+\frac{\partial \hat{\boldsymbol{G}}_{c}}{\partial \eta}+\frac{\partial \hat{\boldsymbol{H}}_{c}}{\partial \zeta}\right)-\frac{1}{\operatorname{Re}_{r e f}}\left(\frac{\partial \hat{\boldsymbol{F}}_{v}}{\partial \xi}+\frac{\partial \hat{\boldsymbol{G}}_{v}}{\partial \eta}+\frac{\partial \hat{\boldsymbol{H}}_{v}}{\partial \zeta}\right)=0 .
$$

In Eq. (1), $\hat{\boldsymbol{Q}}$ is the conservative variable, $\hat{\boldsymbol{F}}_{c}, \hat{\boldsymbol{G}}_{c}$ and $\hat{\boldsymbol{H}}_{c}$ are the inviscid flux terms, and $\hat{\boldsymbol{F}}_{v}, \hat{\boldsymbol{G}}_{v}$ and $\hat{\boldsymbol{H}}_{v}$ are the viscid flux terms along the $\xi, \eta$ and $\zeta$ direction respectively. $R e_{r e f}$ is the reference Reynolds number.

\subsection{Numerical approach}

In order to solve the Navier-Stokes equations, a common solution is to discretize and linearize the equations. For spatial discretization, we use a cell centered finite volume method. The inviscid flux terms are discretized by employing Roe $^{14}$ scheme and a MUSCL ${ }^{15}$ method (Monotone Upstream-centered Schemes for Conservation Laws) can be used to obtain second order accuracy. The viscid flux terms are discretized by using 2order central scheme. After spatial discretization, Eq. (1) can be written as follows: 


$$
\begin{aligned}
& {\left[\boldsymbol{I}+\omega\left(\boldsymbol{A}_{i-1}+\boldsymbol{A}_{i}+\boldsymbol{A}_{i+1}\right)\right]\left[\boldsymbol{I}+\omega\left(\boldsymbol{B}_{j-1}+\boldsymbol{B}_{j}+\boldsymbol{B}_{j+1}\right)\right]} \\
& {\left[\boldsymbol{I}+\omega\left(\boldsymbol{C}_{k-1}+\boldsymbol{C}_{k}+\boldsymbol{C}_{k+1}\right)\right] \Delta \hat{\boldsymbol{Q}}^{n}=\omega \boldsymbol{R} \boldsymbol{H} \boldsymbol{S}} \\
& \Delta \hat{\boldsymbol{Q}}^{n}=\hat{\boldsymbol{Q}}^{n+1}-\hat{\boldsymbol{Q}}^{n} \\
& \boldsymbol{R} \boldsymbol{H} \boldsymbol{S}=\left(\frac{\partial \hat{\boldsymbol{F}}_{c}}{\partial \xi}+\frac{\partial \hat{\boldsymbol{G}}_{c}}{\partial \eta}+\frac{\partial \hat{\boldsymbol{H}}_{c}}{\partial \zeta}\right)^{n}-\frac{1}{R \boldsymbol{e}_{r e f}}\left(\frac{\partial \hat{\boldsymbol{F}}_{v}}{\partial \xi}+\frac{\partial \hat{\boldsymbol{G}}_{v}}{\partial \eta}+\frac{\partial \hat{\boldsymbol{H}}_{v}}{\partial \zeta}\right)^{n}
\end{aligned}
$$

where $\boldsymbol{I}$ is the identity matrix, $\boldsymbol{A}, \boldsymbol{B}$ and $\boldsymbol{C}$ are the Jacobi matrix along the $x, y$ and $Z$ direction respectively $\boldsymbol{R H S}$ is short for right hand side and includes inviscid calculation and viscous calculation.

Eq. (2) then is integrated using ADI scheme ${ }^{16}$ in three sub-steps. At the first sub-step, Eq. (2) is solved in $x$ direction:

$$
\begin{aligned}
& {\left[\boldsymbol{I}+\omega\left(\boldsymbol{B}_{j-1}+\boldsymbol{B}_{j}+\boldsymbol{B}_{j+1}\right)\right]\left[\boldsymbol{I}+\omega\left(\boldsymbol{C}_{k-1}+\boldsymbol{C}_{k}+\boldsymbol{C}_{k+1}\right)\right] \Delta \hat{\boldsymbol{Q}}^{n}=\Delta \hat{\boldsymbol{Q}}^{*}} \\
& {\left[\boldsymbol{I}+\omega\left(\boldsymbol{A}_{i-1}+\boldsymbol{A}_{i}+\boldsymbol{A}_{i+1}\right)\right] \Delta \hat{\boldsymbol{Q}}^{*}=\omega \boldsymbol{R H S}}
\end{aligned}
$$

After LU (Lower-Upper) decomposition method is used to calculate Eq. (3), $\Delta \hat{\boldsymbol{Q}}^{*}$ is obtained. For the second sub-step, Eq. (3) is solved in $y$ direction:

$$
\begin{aligned}
& {\left[\boldsymbol{I}+\omega\left(\boldsymbol{C}_{k-1}+\boldsymbol{C}_{k}+\boldsymbol{C}_{k+1}\right)\right] \Delta \hat{\boldsymbol{Q}}^{n}=\Delta \hat{\boldsymbol{Q}}^{* *}} \\
& {\left[\boldsymbol{I}+\omega\left(\boldsymbol{B}_{j-1}+\boldsymbol{B}_{j}+\boldsymbol{B}_{j+1}\right)\right] \Delta \hat{\boldsymbol{Q}}^{* *}=\Delta \hat{\boldsymbol{Q}}^{*}}
\end{aligned}
$$

Eq. (4) is adopted the same LU decomposition method, $\Delta \hat{\mathbf{Q}}^{* *}$ is got. For the third substep, Eq. (4) is solved in $z$ direction:

$$
\left[\boldsymbol{I}+\omega\left(\boldsymbol{C}_{k-1}+\boldsymbol{C}_{k}+\boldsymbol{C}_{k+1}\right)\right] \Delta \hat{\boldsymbol{Q}}^{n}=\Delta \hat{\boldsymbol{Q}}^{* *} .
$$

Similarly, Eq. (5) is used LU decomposition method, $\Delta \hat{\boldsymbol{Q}}^{n}$ finally is obtained.

For ADI scheme used in this paper, each numerical step is split into three sub-steps based on $x, y$ and $z$ direction of the whole solution domain, and the linear equation is solved implicitly in one direction while processing the relevant calculation in the other directions explicitly.

\section{CUDA Implementation}

Traditionally, the multi-block, structured grid CFD parallel computing on CPUs is based on domain decomposition using MPI and OpenMP programming, in which each CPU core processes one or more mesh blocks separately. However, there is a big difference between CPU and GPU architectures. GPUs can execute a large number of threads concurrently and hide memory latency by efficient switching from one thread to another. So the design principle of GPU parallel algorithm is to make full use of millions of lightweight threads on the GPUs. Considering CUDA programming features and data 
dependencies of ADI solver, we propose two fine-grain schemes, namely "one-threadone-point" and "one-thread-one-line", to maximize the GPU performance.

\subsection{RHS parallel algorithm based one-thread-one-point scheme}

For the RHS calculation, there is no data dependence. In order to maximize parallelism and utilize lightweight threads on the GPUs as many as possible, we propose this onethread-one-point scheme. The challenge of this approach is getting the corresponding relation between the GPU threadID and cell, and calculating the boundary condition correctly is also very important. The details of one-thread-one-point scheme are shown in Fig. 1.

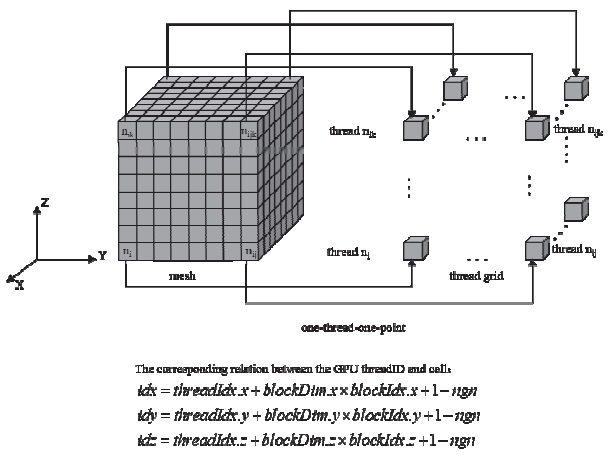

Fig. 1. For the one-thread-one-point scheme, 3D mesh cells on $x, y$ and $z$ directions are mapped to GPU threads grid on $x, y$ and $z$ directions. $i d x$, idy and $i d z$ are the index of GPU threadID on $x, y$ and $z$ directions, respectively. ngn denotes the layer number of ghost cell on the boundary.

\subsection{ADI parallel algorithm based one-thread-one-line scheme}

According to the calculation process of ADI scheme, the variable increments, $\Delta \hat{\boldsymbol{Q}}^{*}, \Delta \hat{\boldsymbol{Q}}^{*}$ and $\Delta \hat{\boldsymbol{Q}}^{n}$ are solved implicitly in $x, y$ and $z$ directions, respectively. Form the data dependencies point of view, each grid line in the same direction can be solved in parallel. So we design a one-thread-one-line scheme to maximize parallelism on the GPUs. Fig. 2 and Fig. 3 show the details of this one-thread-one-line scheme.
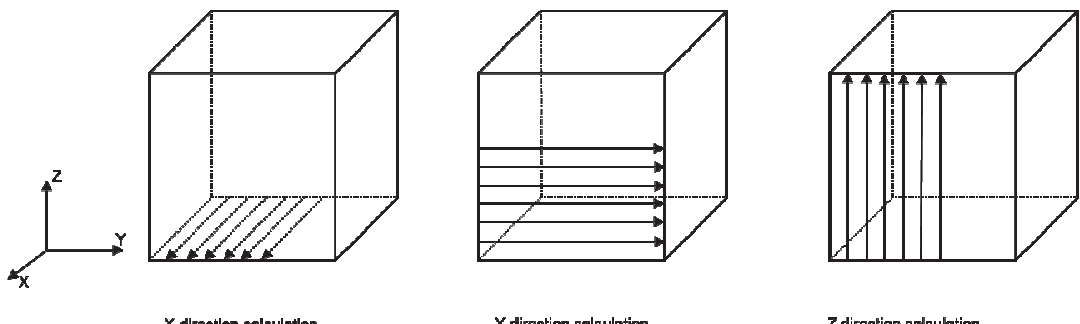

$X$ diraction calculation

Y direction calculation

$Z$ direction calculetion

Fig. 2. The calculation of ADI scheme in $x, y$ and $Z$ directions. 


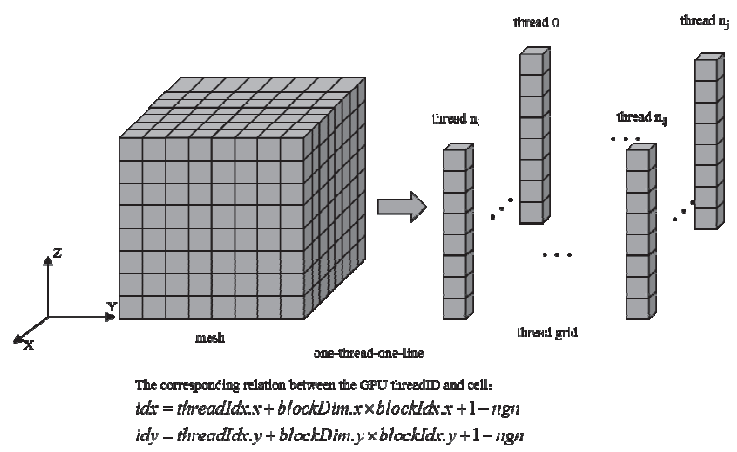

Fig. 3. For the one-thread-one-line scheme, each CUDA thread calculates all cell on the $Z$ direction of 3D mesh. idx and idy are the index of GPU threadID on $x$ and $y$ directions, respectively. ngn denotes the layer number of ghost cell on the boundary.

\subsection{Residual calculation based parallel reduction}

For calculation residual value during CFD simulation, it is usually needed that specific values are summed over a large array. This summation process is easy to implement in CUDA, but there is a need of global synchronization to get it right. Since the CUDA model is lack of efficient global synchronization mechanism, we use a method knows as parallel reduction ${ }^{17}$ to support the fine-grain parallelization. The detail of parallel reduction algorithm is shown in Fig. 4.

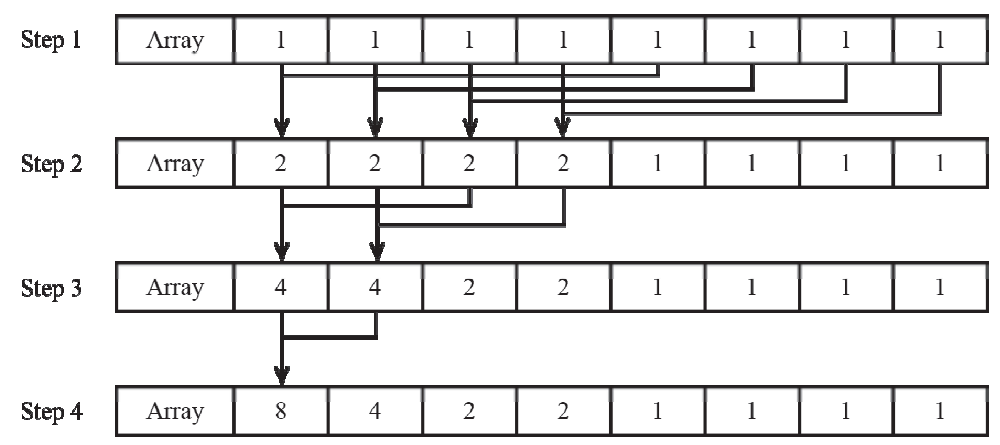

Fig. 4. Simplified parallel reduction algorithm for the residual calculation

After the above methods are implemented, the procedure of a full GPU version of the ADI solver is illustrated as Fig. 5. In order to remove a lot of redundant data transfers between CPU and GPU, there are only two CPU/GPU data transfers, one is from CPU to GPU, and the other is form GPU to CPU. Almost all of ADI solver is calculated on the GPUs, the CPU only executes boundary information exchange and termination condition judgment.

After the solving step, the post-processing visualizes flow and analyzes the result of outputs. 


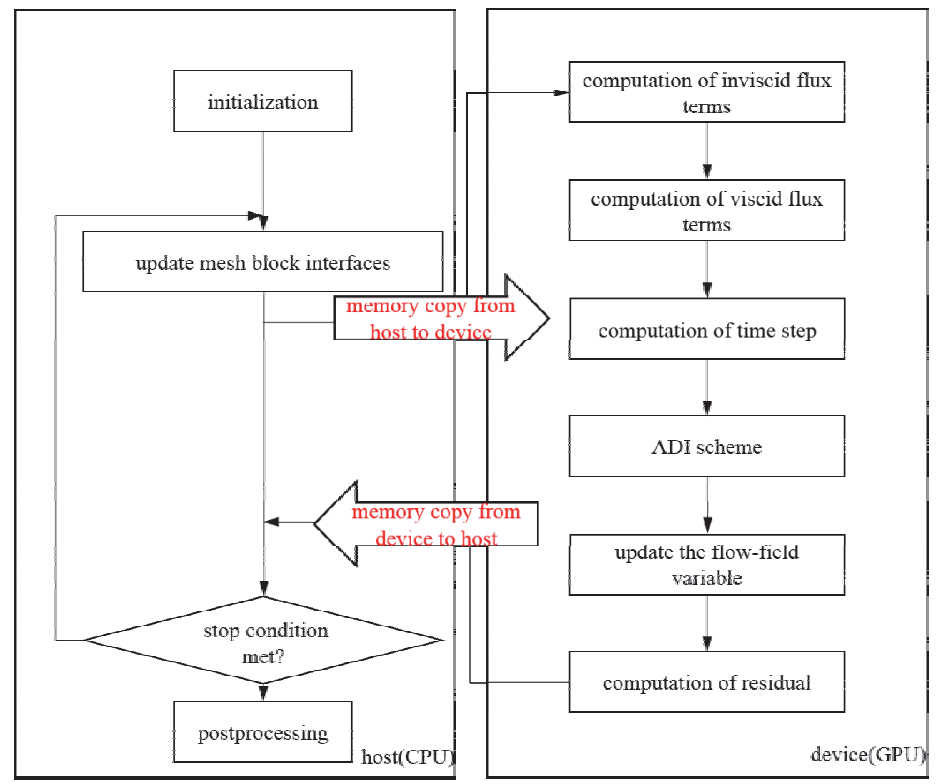

Fig. 5. Flow chart of the ADI solver.

\section{OpenMP-CUDA Implementation}

Computing resources in CPU/GPU heterogeneous system include multi-core CPUs and many-core GPUs. In Section 3, CPUs and GPUs cooperate as the way that GPUs perform arithmetic operations and CPUs only manage GPU computation and communication. This is obviously a vast waste of the computational capability of CPUs. Therefore, to tap the full potential of heterogeneous platform and maximize the performance, it is vital to collaborate CPUs and GPUs for CFD simulation.

By using the CPU/GPU collaborative model, we present a dual-level OpenMPCUDA parallelization scheme that is merging parallelism on the CPUs using OpenMP with parallelism on the GPUs using CUDA. Fig. 6 illustrates the CPU/GPU cooperative model as well as the pseudocode of CPU/GPU cooperative parallel algorithm. Our research is based on a GPU, but this model is not limited to a GPU. 

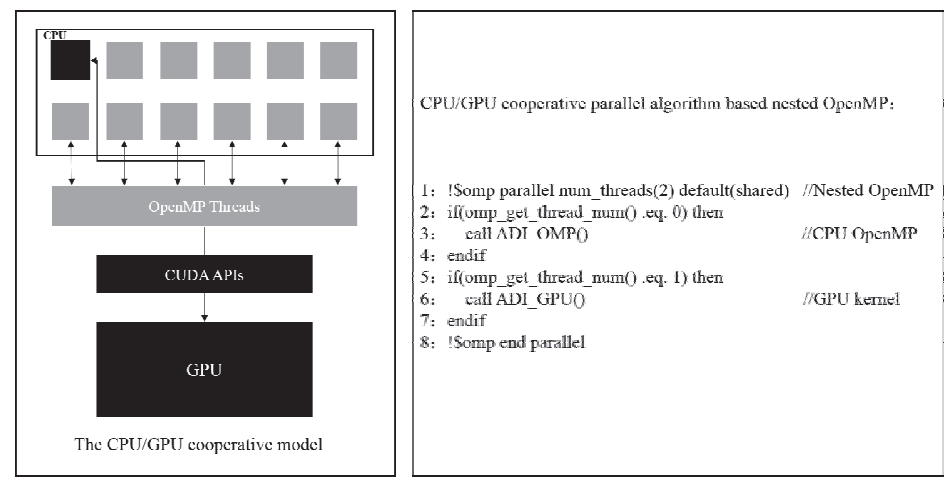

Fig. 6. The CPU/GPU cooperative model and pseudocode illustrating CPU/GPU cooperative parallel algorithm.

To implement a collaborative multi-block ADI solver, we develop two different versions of ADI solver, the CPU version ADI_OMP parallelized with OpenMP and the GPU version ADI_GPU parallelized with CUDA threads. We create two OpenMP threads that is thread 0 and thread 1 at the first-level parallel region. Thread 0 calls ADI_OMP to deal with the CPU-computed grid blocks using the second-level nested OpenMP threads. Thread 1 calls ADI_GPU to deal with GPU-computed grid blocks using CUDA threads. During collaboration, CPUs and GPUs are concurrently working on their own grid blocks.

\section{MPI-OpenMP-CUDA Implementation}

Considering the fact that memory on a single node becomes inadequate when the simulation size grows, we use MPI to increase the simulation size on multi-nodes ${ }^{18}$. Therefore, a tri-level hybrid programming pattern MPI-OpenMP-CUDA that merges fine-grain parallelism using OpenMP and CUDA threads with coarse-grain parallelism using MPI for inter-node communication is presented. Fig. 7 illustrates the MPIOpenMP-CUDA programming pattern.

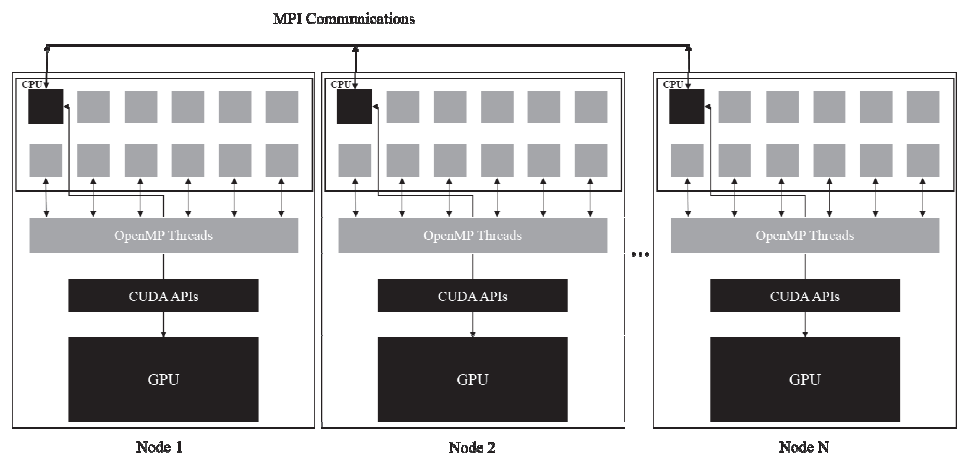

Fig. 7. The MPI-OpenMP-CUDA programming pattern. Collaborating intra-node CPU and GPU, exchanging inter-node data via MPI communication. 
However, there are no CUDA commands to operate data transfers between GPUs in different nodes ${ }^{18}$. The data transfers needs CPU-side data buffering, and then exchange by using MPI. Fig. 8 illustrates the process of data transfers between different nodes.

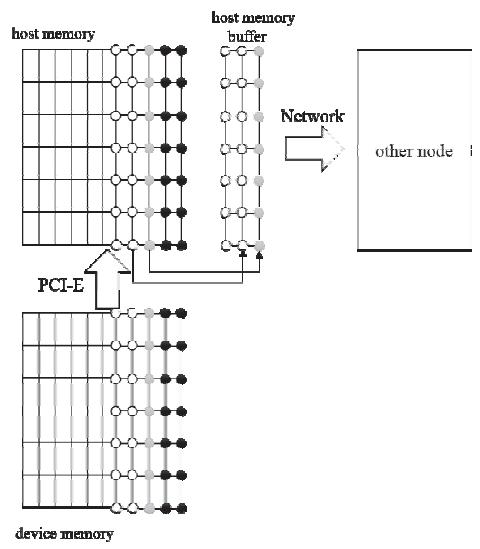

Fig. 8. Data exchange with the buffering the boundaries data method. The black cells means ghost cells, the white cells means boundary cells, and the grey cells means the interface meshes.

Due to the PCI-E bus bandwidth between CPU and GPU and the network bandwidth among compute nodes are much lower than the bandwidth between devices and its own main memory, designing a transfer strategy to overlap the computation and communication is very necessary. In this paper, we propose a strategy by using nonblocking MPI communication, CUDA multiple streams and the asynchronous data transfer mechanism between CPU and GPU. Fig. 9 shows this strategy in detail. There are five stages of software pipeline: the whole data of a grid block transfer from CPU to GPU, CUDA kernel performed on GPU, the data transfers from GPU to CPU, the data packing on CPU, and data transfers to other nodes using non-blocking MPI communication.

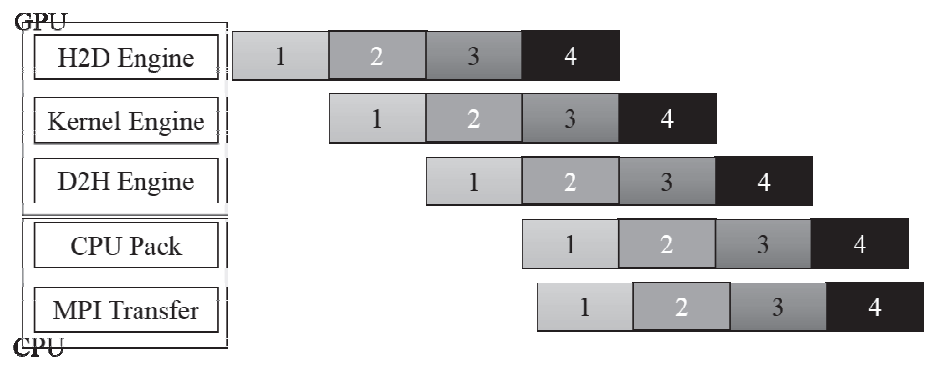

Fig. 9. Overlapping the collaborative CPU/GPU computation and communication. 


\section{Deng et al.}

\section{Experimental Results}

In this section, we first implement the ADI solver on a heterogeneous platform. And then we adopt a numerical experiment to validate the correctness and accuracy of GPU solver. Finally the performance is evaluated with the aforementioned implementation.

\subsection{Implementation}

The solver which is 2 th order accurate and advances in time with ADI scheme, is carried on a CPU/GPU heterogeneous platform. A single node of this platform consists of Two Intel Xeon X5670 CPUs and one Nvidia Tesla M2050 GPU. The PGI compiler 2012 release which supports Fortran, CUDA languages, MPI parallelism and nested OpenMP parallelism, is used. The details of hardware and software are shown in Table 1. The GPU code is implemented using CUDA Fortran. All the code is compiled with -O3 option. Double precision is used in all computations.

Table 1. Platform configurations.

\begin{tabular}{lll}
\hline & GPU & CPU \\
\hline Units per node & 1 & 2 \\
Cores per node & 448 & 12 \\
Type & Nvidia Tesla M2050 & Intel Xeon X5670 \\
Memory & about 3 GB & $48 \mathrm{~GB}$ \\
Interconnect & PCI-E 2.0 & Fat tree network \\
Price & \$1350.00 & $\$(1128.75 \times 2)$ \\
Power consumption & 225 W & $(95 \times 2) \mathrm{W}$ \\
Software stack & CUDA 4.0 & \\
\hline
\end{tabular}

In accordance with the access properties and size of data structures in CFD applications, it can be divided into three types: flow variable, flow parameter and standard value. The flow variable is usually large multi-dimensional arrays and it need to be performed read and write operations multiple times. The flow parameter is generally scalar variables and it remains the same in the computing process. The standard value is read-only global scalar variables in general and it is defined as constant in the program. To improve the performance of CFD application, it is very important to make full use of the GPU's memory hierarchy. Therefore, based on the characteristics of data structures in CFD applications, flow parameter and standard value are stored in constant memory to reduce the data transfers between CPU and GPU, and flow variable is stored in global memory to benefit the data exchanges between CPU and GPU. All data structures are stored contiguously for one dimension which is good for coalescing global memory accesses.

\subsection{Numerical experiments}

A test case is the solution for the steady flow around NACA0012 airfoil at a Mach number of 0.3 , the Reynolds number is $1.86 \times 10^{6}$, the angle of attack is $3.59^{\circ}$. Fig. 10 shows the grid structure. The NACA0012 airfoil configuration used in this work contains 
120,000 cells. The residual and pressure is illustrated in Fig. 11. The CPU version and the GPU version obtain the same results.

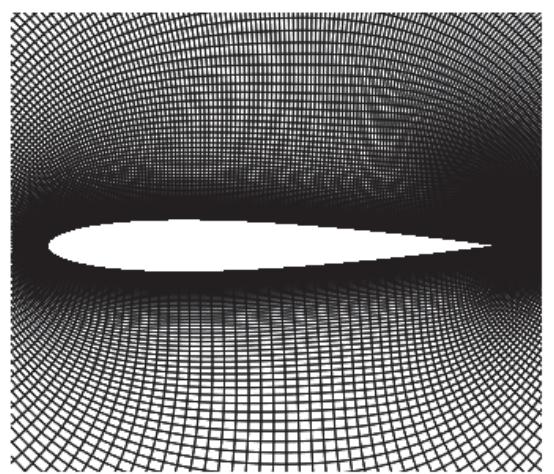

Fig. 10. The grid structure of NACA0012.

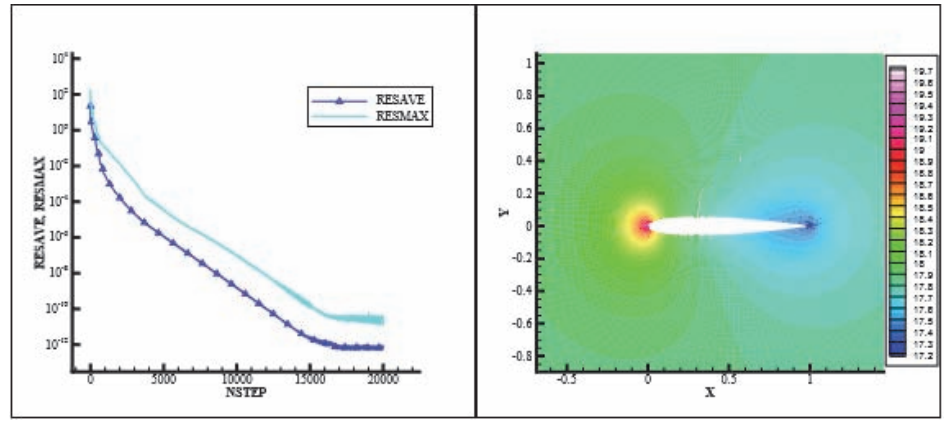

Fig. 11. The residual and the contour of the pressure.

\subsection{Single GPU performance}

A test case is the solution for the steady flow around a circular cylinder. The incoming Mach number is 1.6 , the Reynolds number is $1.1 \times 10^{7}$, the angle of attack is $0^{\circ}$. The benchmark simulations use three kinds of structured grid: $128 \times 128 \times 64,128 \times 128 \times 96$ and $128 \times 128 \times 128$ and the grid are all divided to 1,2 and 4 blocks, respectively. Fig. 12 shows the speedup of a M2050 GPU over a single core of X5670 CPU, the speedup of a M2050 GPU over a six-core X5670 CPU using 6 OpenMP threads and dual six-core X5670 CPUs using 12 OpenMP threads. The result shows that the computational performance on a M2050 is about $13.4 \times$ faster than on a single core of X5670 CPU and is also $6.0 \times$ speedup over dual six-core X5670 CPUs when the grid size is $128 \times 128 \times 128$. A speedup is about 11.1 when comparing the price/performance ratio of M2050 GPU to the price/performance ratio of dual X5670 CPUs. We observe slight performance degradation for both CPU and GPU when the block number is increased. This is caused by incurring extra OpenMP and CPU/GPU overheads. In Fig. 13 shows the performance results for different grid sizes with four blocks. The larger problem sizes, the better 
performance. This is because higher workloads can better overlap the computation and global memory access for GPUs.

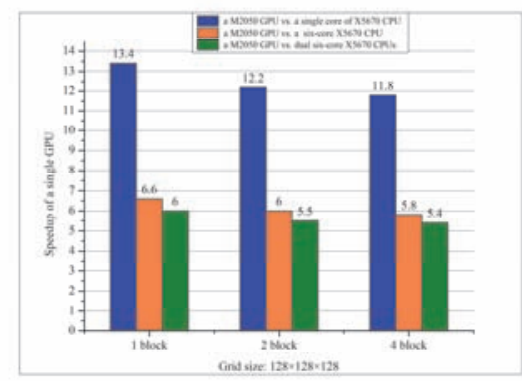

Fig. 12. Single GPU speedup for fixed grid size.

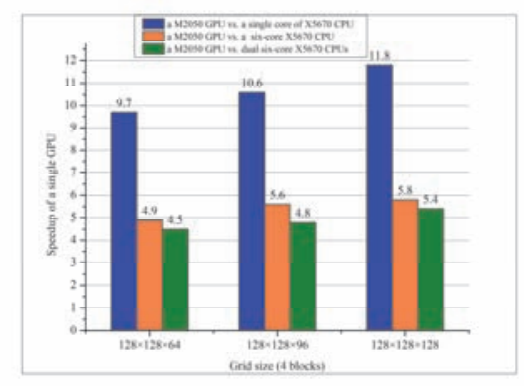

Fig. 13. Single GPU speedup for different grid sizes.

\subsection{Single node performance}

Also, a test case is the solution for the steady flow around a circular cylinder. The benchmark simulations use three kinds of structured grid: $128 \times 128 \times 64,128 \times 128 \times 96$ and $128 \times 128 \times 128$ and the grid are all divided to four blocks. Fig. 14 shows the runtime per iteration for the three grid sizes while the workload on GPU varying. Experimental results show that the computational performance of $128 \times 128 \times 64$ and $128 \times 128 \times 96$ on a single node are $1.06 \times$ and $1.35 \times$ speedup relative to a naïve GPU-only approach, respectively. However, the performance of a naïve GPU-only approach is better when the gird size is $128 \times 128 \times 128$.

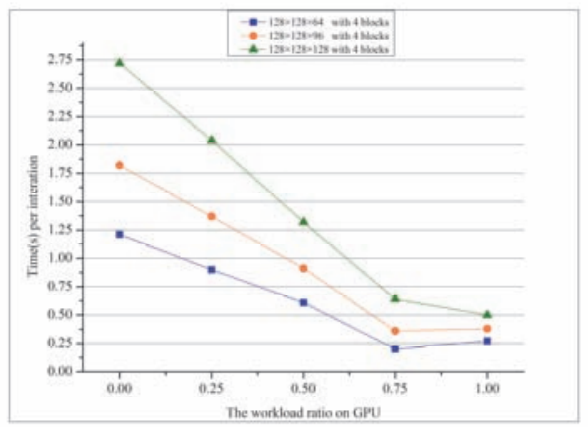

Fig. 14. Performance comparison with varying workload on GPU.

\subsection{Multi-node performance}

In order to test the parallel performance, measurements are performed for both strong scaling and weak scaling efficiency.

The strong scaling evaluation in which the dataset size remains fixed as the number of nodes increases is presented in Fig. 15. The total grid sizes are fixed as $128 \times 128 \times 64$, 
$128 \times 128 \times 96$ and $128 \times 128 \times 128$. We use the performance achieved on one node as a baseline. The computational performance on four nodes performs $3.76 \times, 3.83 \times$ and $3.87 \times$ speedup over the baseline, respectively.

The weak scaling efficiency evaluation in which the dataset size and the number of nodes increase proportionally is presented in Fig. 16. For the grid size $128 \times 128 \times 64$ on each node, the efficiency drops from $100 \%$ with one node to $71 \%$ with four nodes. For the grid size $128 \times 128 \times 96$ on each node, the efficiency drops from $100 \%$ with one node to $73 \%$ with four nodes. For the grid size $128 \times 128 \times 128$ on each node, the efficiency drops from $100 \%$ with one node to $76 \%$ with four nodes.

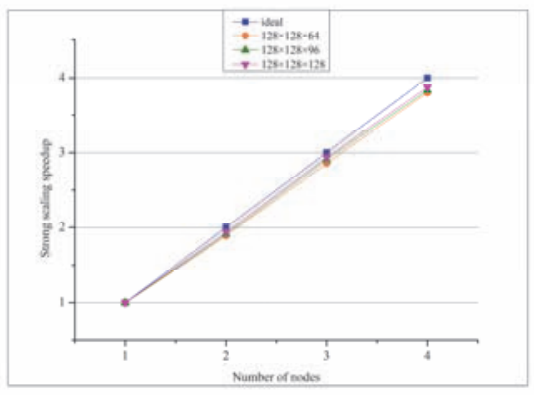

Fig. 15. Strong scaling speedup on heterogeneous platform. The one node results are used as the baseline.

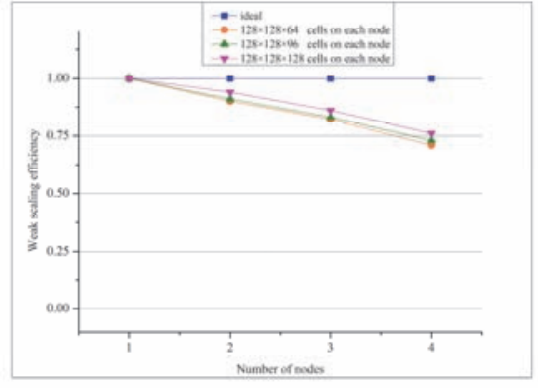

Fig. 16. Weak scaling efficiency on heterogeneous platform. The one node results are used as the baseline.

\section{Conclusions and Future work}

In this paper, a dual-level and tri-level parallel implementations of a three-dimensional, implicit, compressible viscous flow solver for multi-block structured grid on heterogeneous platform by using MPI, OpenMP and CUDA, are developed. We presented some novel techniques to maximize the performance and implemented CPUGPU collaboration using the CPU/GPU collaborative model. And we also propose a strategy to overlap the computation with communication using non-blocking MPI communication, CUDA multiple streams and the asynchronous data transfer mechanism between CPU and GPU. The performance measurements demonstrate that an implicit, multi-block compressible Navier-Stokes solver can achieve significant performance improvement on heterogeneous platform.

For future work, we are planning to implement an auto-tuing infrastructure for multiblock structured grid CFD simulations on heterogeneous systems.

\section{References}

1. Levesque J M. Application Development for Titan-A Multi-Petaflop Hybrid-Multicore MPP System[C]//High Performance Computing, Networking, Storage and Analysis (SCC), 2012 SC Companion: IEEE, 2012: 1731-1821. 


\section{Deng et al.}

2. Yang X J, Liao X K, Lu K, et al. The TianHe-1A supercomputer: its hardware and software[J]. Journal of Computer Science and Technology, 2011, 26(3): 344-351

3. Xu C, Deng X, Zhang L, et al. Parallelizing a High-Order CFD Software for 3D, Multiblock, Structural Grids on the TianHe-1A Supercomputer[J]. Lecture Notes in Computer Science, 2013.

4. Corrigan A, Camelli F, Löhner R, et al. Running unstructured grid based CFD solvers on modern graphics hardware[J]. AIAA paper, 2009, 4001: 22-25.

5. Jespersen D C. Acceleration of a CFD code with a GPU[J]. Scientific Programming, 2010, 18(3-4): 193-201.

6. Griebel M, Zaspel P. A multi-GPU accelerated solver for the three-dimensional two-phase incompressible Navier-Stokes equations[J]. Computer Science-Research and Development, 2010, 25(1-2): 65-73.

7. Jacobsen D A, Thibault J C, Senocak I. An MPI-CUDA implementation for massively parallel incompressible flow computations on multi-GPU clusters[C]//48th AIAA Aerospace Sciences Meeting and Exhibit. 2010, 16.

8. Jacobsen D A, Senocak I. Scalability of incompressible flow computations on multi-GPU clusters using dual-level and tri-level parallelism [C]//49th AIAA Aerospace Sciences Meeting including the New Horizons Forum and Aerospace Exposition, vol. 4, AIAA, 2011-947.

9. $\mathrm{Xu} \mathrm{C}$, Deng X, Zhang L, et al. Collaborating CPU and GPU for large-scale high-order CFD simulations with complex grids on the TianHe-1A supercomputer[J]. Journal of Computational Physics, 2014, 278: 275-297.

10. Sakharnykh N. Tridiagonal solvers on the GPU and applications to fluid simulation[C]//NVIDIA GPU Technology Conference. 2009.

11. Sakharnykh N. Efficient tridiagonal solvers for ADI methods and fluid simulation[C]/NVIDIA GPU Technology Conference. 2010.

12. Wei Z, Jang B, Zhang Y, et al. Parallelizing alternating direction implicit solver on GPUs[J]. Procedia Computer Science, 2013, 18: 389-398.

13. Wang Y X, Zhang L L, Liu W, et al. Efficient parallel implementation of large scale 3D structured grid CFD applications on the Tianhe-1A supercomputer[J]. Computers \& Fluids, 2013, 80: 244-250.

14. Toro E F. Riemann solvers and numerical methods for fluid dynamics. Springer Verlag, Berlin, 1997

15. Van Leer B. Towards the ultimate conservative difference scheme. V. A second-order sequel to Godunov's method[J]. Journal of computational Physics, 1979, 32(1): 101-136.

16. Peaceman D W, Rachford, Jr H H. The numerical solution of parabolic and elliptic differential equations[J]. Journal of the Society for Industrial \& Applied Mathematics, 1955, 3(1): 28-41.

17. Lu F, Song J, Cao X, et al. Acceleration for CFD applications on large GPU clusters: an NPB case study[C]//Computer Sciences and Convergence Information Technology (ICCIT), 2011 6th International Conference on. IEEE, 2011: 534-538.

18. Cao W, Xu C, Wang Z, et al. CPU/GPU computing for a multi-block structured grid based high-order flow solver on a large heterogeneous system[J]. Cluster Computing, 2014, 17(2): 255-270.

19. Ruetsch G, Fatica M. CUDA Fortran for scientists and engineers[J]. NVIDIA Corporation, 2011, 2701.

20. Harris M. Optimizing parallel reduction in CUDA[J]. NVIDIA Developer Technology, 2007, 2(4). 